\title{
Place Type Code
}

National Cancer Institute

\section{Source}

National Cancer Institute. Place Type Code. NCI Thesaurus. Code C93994.

A coded value specifying the kind of place. 